\title{
Splitting of the middle layer of LPW SAFNWC/MSG satellite product in order to improve the monitoring of pre-convective environments
}

\author{
G. Cuevas, M. A. Martinez, M. Velazquez, J. Ruiz, and M. Manso \\ Agencia Estatal de Meteorología (former INM), Spain
}

Received: 31 December 2007 - Revised: 5 May 2008 - Accepted: 15 May 2008 - Published: 23 May 2008

\begin{abstract}
Seven of the infrared channels from the Spinning Enhanced Visible and Infrared Imagery (SEVIRI) instrument, on board the Meteosat Second Generation (MSG), are used to retrieve Layer Precipitable Water (LPW) and Stability Analysis Imagery (SAI) in the SAFNWC framework. Both products are retrieved using a statistical retrieval based on neural networks; they are routinely generated every fifteen minutes at a satellite horizontal resolution of $3 \mathrm{~km}$ in NADIR only in cloud-free areas.

Many factors are involved in the development of severe weather and these parameters are only some of the indicators. However, due to the high resolution of these products, the use of them in conjunction with satellite and radar images can help to identify mesoscale features related to convection. The MSG moisture and parcel instability time trend fields are especially useful during the period previous to convection. Once the outbreak of convection occurs, the products calculated in the clear air pixels surrounding the convective system can give us hints to anticipate its evolution.

SAFNWC LPW and SAI were analyzed for a severe weather event during August 2004. A thunderstorm over Teruel (Spain) produced intense precipitation and hail; a tornado developed while this thunderstorm was moving towards SE. The pre-convective parcel potential buoyancy and moisture SAFNWC products changed in a way that was consistent with the observed intense convective activity. In previous studies, the atmospheric moisture in medium levels, which has been proven to be relevant in some cases, was represented by only one level parameter (ML: middle layer LPW). However, it was observed that this layer is too thick to do an adequate analysis of moisture available for convection. Hence, an improvement on the LPW algorithm has been carried out by splitting the middle layer into two new sub-layers (approximately separated at $700 \mathrm{hPa}$ ) and training two new neural networks. The impact of monitoring moisture in the new sub-layers separately in this severe weather event has been tested, and the improvements achieved have been evaluated.
\end{abstract}

\section{Introduction}

This work is embedded in the EUMETSAT Satellite Application Facilities for Nowcasting and Very Short Range Forecasting (NWCSAF). The aim is to develop and distribute software which provides products for nowcasting in near real time (every $15 \mathrm{~min}$ ) at full horizontal resolution. In this framework, seven of the infrared channels from the Spinning Enhanced Visible and Infrared Imagery (SEVIRI) instrument, on board the Meteosat Second Generation (MSG), are

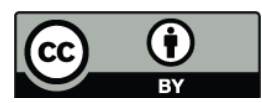

Correspondence to: G. Cuevas (gcuevas@inm.es) used to retrieve the PGE07 Layer Precipitable Water (LPW) and the PGE08 Stability Analysis Imagery (SAI) products only in cloud-free areas. PGE01 Cloud Mask product is used as input in order to identify clear and cloudy pixels. Both products are retrieved using a statistical retrieval algorithm based on neural networks. Details on the architecture and training dataset are described in Martínez (2008).

The LPW product provides information on the water vapour contained in a vertical column of unit cross-section area for three layers across the troposphere (low, middle and high) and in the total layer. The SAI product, in particular the lifted index (LI), gives estimations of the parcel instability. Both products are only retrieved in cloud-free areas. They are only some of the indicators of severe weather potential

Published by Copernicus Publications. 

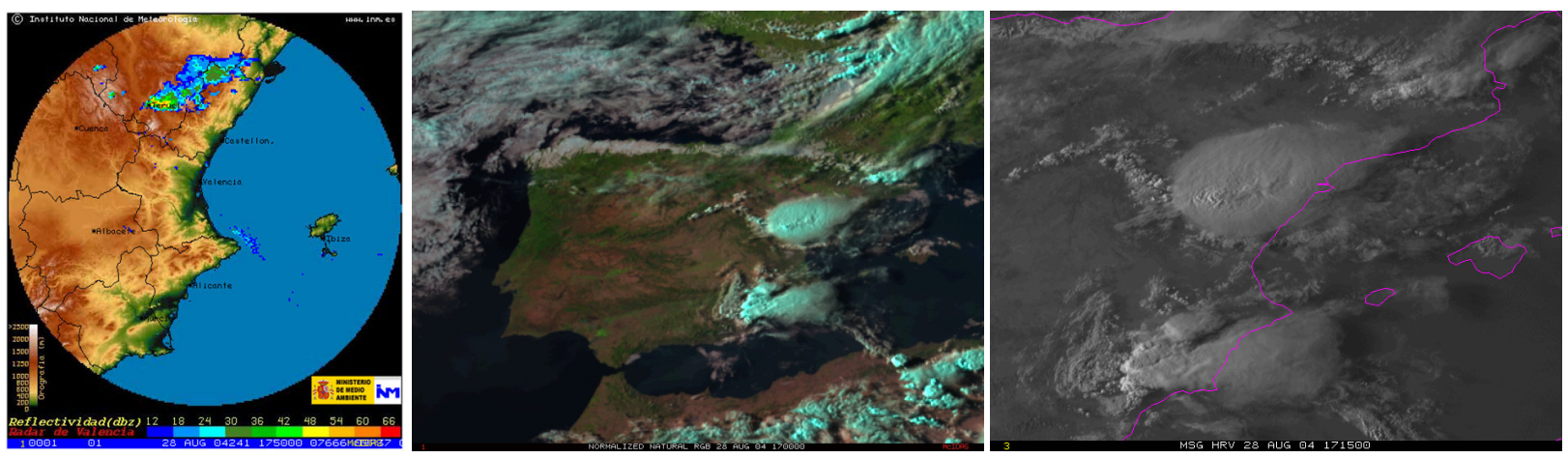

Figure 1. Zaragoza radar image at 17:50 (left); SEVIRI Natural RGB (middle) and High Resolution Visible (right), both at 17:30.

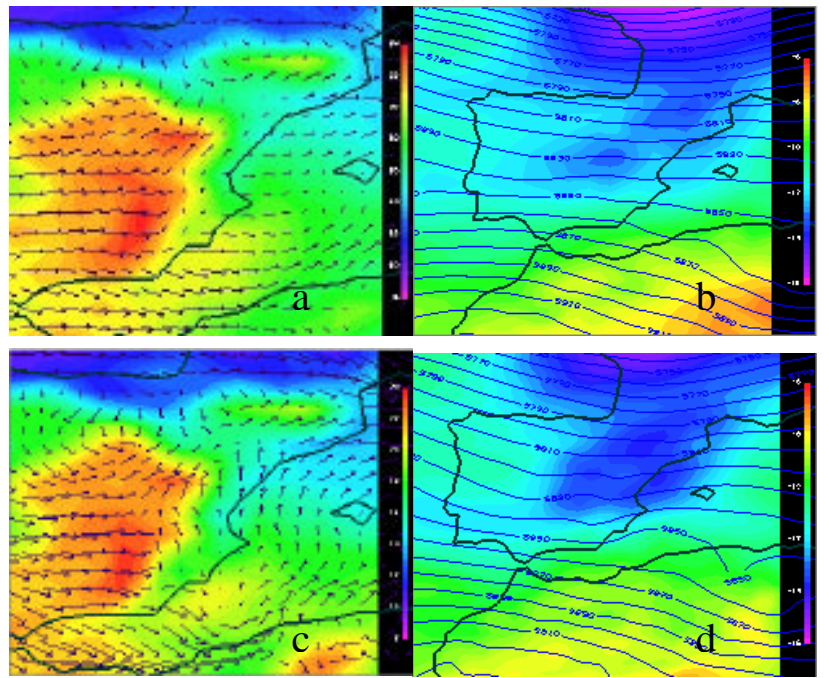

Figure 2. ECMWF model $00 \mathrm{Z}+15$ forecast: temperature and wind fields at $850 \mathrm{hPa}(\mathbf{a}), 00 \mathrm{Z}+15$ temperature and geopotential at $500 \mathrm{hPa}(\mathbf{b})$, and similar fields for the $12 \mathrm{Z}+3$ forecast (c and d).

since many factors are involved in the onset of deep convection, but they have the advantage of their high spatial and temporal resolution. Along with satellite and radar imageries they may help to locate mesoscale features related to convection.

The MSG moisture and parcel instability time trend fields are very useful during the period preceding the outbreak of convection. In particular, the LPW parameter at middle levels (ML) has proved to be an important ingredient in some previously studied convective weather events. However, as shown in a previous paper (Martínez, 2007), this layer is too thick $(840 \mathrm{hPa}-437 \mathrm{hPa})$ to perform an adequate monitoring of pre-convective precipitable water relevant for upcoming intense convective activity. Hence, it was carried out a splitting of the middle layer into two new sub-layers separated at approximately $700 \mathrm{hPa}$ by training two new neu- ral networks. The behaviour of the new sub-layers in this severe weather event was tested, together with the conventional clear-air products. The improvements obtained with these new sub-layers in the monitoring of the pre-convective environment are evaluated.

\section{Case study and synoptic analysis}

The behaviour of these new sub-layers was tested (along with LPW and SAI operational products) in a particular severe weather event. A severe weather episode occurred between 17:00-19:00 Z on the 28 August 2004 at Northeast of Spain. A thunderstorm of intense precipitation and hail over Teruel was reported. A tornado developed as this thunderstorm was moving towards Southeast. A descriptive report of the episode was done by the INM (Conesa, 2004).

The tornado took place between 17:00 and 17:20 Z, crossing an area of a considerable elevation (1300-1400 m a.s.1.); during this period, supercell signatures were observed by the INM Zaragoza radar. Only reflectivity information is available since the area was outside the Doppler radar coverage. Then, a distinct radar mesocyclone signature could not be observed, but it may be discerned in the MSG available imagery.

Most supercell tornadoes form below the middle or low level mesocyclone. If a moderate supply of warm air is available ahead of the storm, it could be tornadic for some time. The mesocyclone draws energy into the storm so that it can last for hours. It can be detected by conventional radar as a hook echo. In the mesocyclone, air is drawn into the storm. It is believed that vertical wind shear increases the potential a thunderstorm will have to develop rotation and therefore to become severe.

METEOSAT images can also help to illustrate this convective event. Even though there is no Doppler Radar Available to confirm it, the anomalous movement of the system to the right of the mean flow, confirmed in satellite imagery loops, made us suspect that rotation was present in the main updraft, and therefore there was a chance for tornadogenesis. 

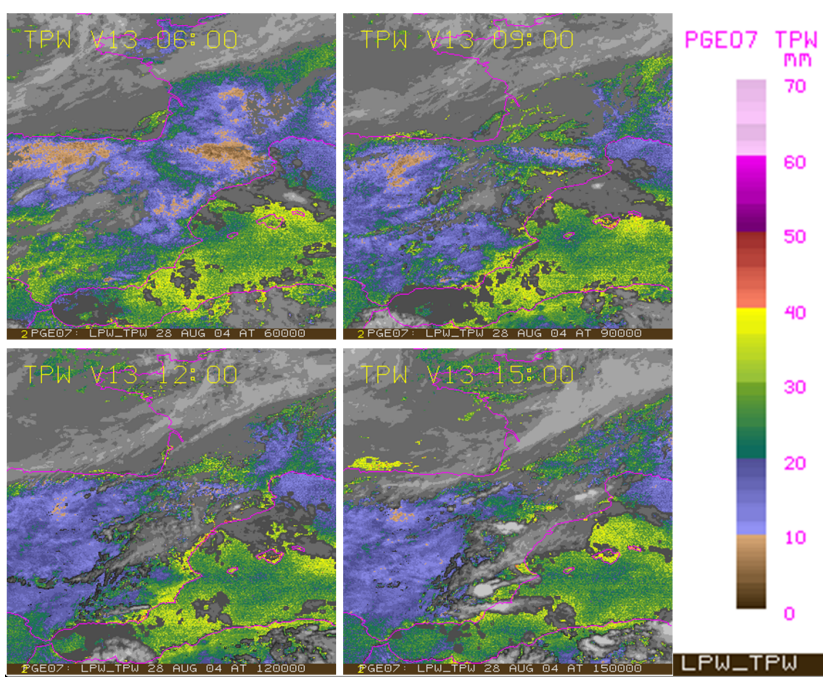

Figure 3. TPW evolution at 06:00, 09:00, 12:00 and 15:00.

Several channels and RGBs proposed by EUMETSAT (natural, air-mass, convective) were qualitatively inspected by means of image loops. (Fig. 1 shows some radar and satellite image samples).

Although this work focuses on the analysis of thermodynamic parameters, the synoptic situation in which the event developed is also described (based on the ECMWF model).

The 00:00 Z ECMWF forecast $(+15 \mathrm{H})$ is of more relevance for the forecasters, because the 12:00 Z forecast was delivered after the development of convection (ECMWF not available in real time, as all NWP models, due to the assimilation window delay). Convergence at low levels and cooling at $500 \mathrm{hPa}$ was already predicted at 00:00 Z (see Fig. 2a and $b$ ). These are common conditions for the onset of convective weather in the area of study. Anyhow, the 12:00 Z ECMWF was also analysed, showing that the synoptic pattern changed, enhancing both effects of convergence and cooling (see Fig. 2c and d)

\section{Analysis of parameters (pre-convective situation)}

The moisture parameters were analysed during the hours before the outbreak of convection using a time sequence of images every $15 \mathrm{~min}$ (visual inspection of image loops). This is the best way to monitor moistening trends. The three moisture layer parameters conventionally used: BL (Bottom Layer), ML (Middle Layer), HL (High Layer) were examined together with the Total Precipitable Water and Lifted Index (time evolution). The low values of LI as well as the increasing TPW can help us to anticipate how intense the convection will be (once the convection outbreaks).

Figures 3 and 4 show some picks of the time evolution loops for TPW and ML in order to appreciate the general environment over the Peninsula.
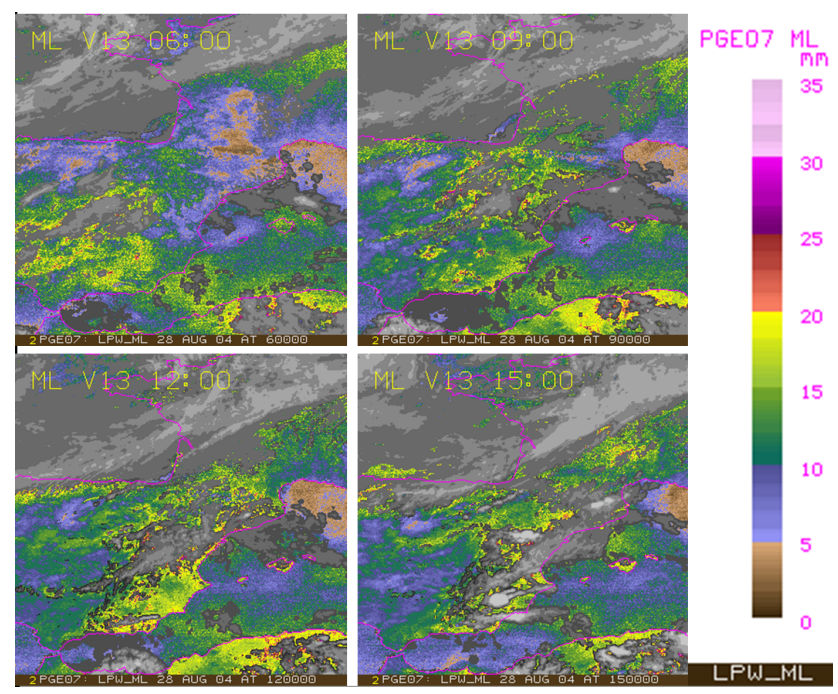

Figure 4. ML evolution at 06:00, 09:00, 12:00 and 15:00 Z.

During the first hours (06:00-09:00), a moisture spot was highlighted on the Southwest of the Iberian Peninsula, while the Northeast presented dry areas. In the image loop it was appreciated that this moisture spot moved on to the NE increasing the humidity at middle and high levels around the region where the thunderstorm under study developed.

Precipitable water increased in all layers, but the ML is the one which contributes the most (see Fig. 4). The behaviour of the two middle sub-layers M1 (low) and M2 (high) was also tested.

Before the outbreak of the severe convection, an increase of water content is observed for both layers. There is a continuous increase in water content for M1 (see Fig. 5), which may be explained by the moisture convergence observed at $850 \mathrm{hPa}$. M2 increase could come from lower levels (see Fig. 6).

\section{Comparison with ECMWF and MODIS}

Finally, a qualitative comparison was carried out between these new parameters M1 and M2 and the equivalent ones generated for the ECWMF forecasts at $00+15 \mathrm{H}$ and $12+3 \mathrm{H}$. It is noticed a significant change on the model forecast from one run to the next (see Fig. 7). The M1 pattern fits better the $12+3 \mathrm{H}$ forecast at the moment previous to convection. Similar results are found for M2 parameter (not shown here) as well as for every other parameter.

Another comparison was done by means of the available MODIS imagery. MODIS TERRA passed at 10:50, and MODIS AQUA passed at 12:30, so the latter was chosen for the comparison at the time before the start of convection. Subsequent images did not already cover the zone of interest.

Figure 8 depicts the M2 parameter from SAF, from the ECMWF (forecast at $00+12 \mathrm{H}$ and analysis at 12:00 Z) and 


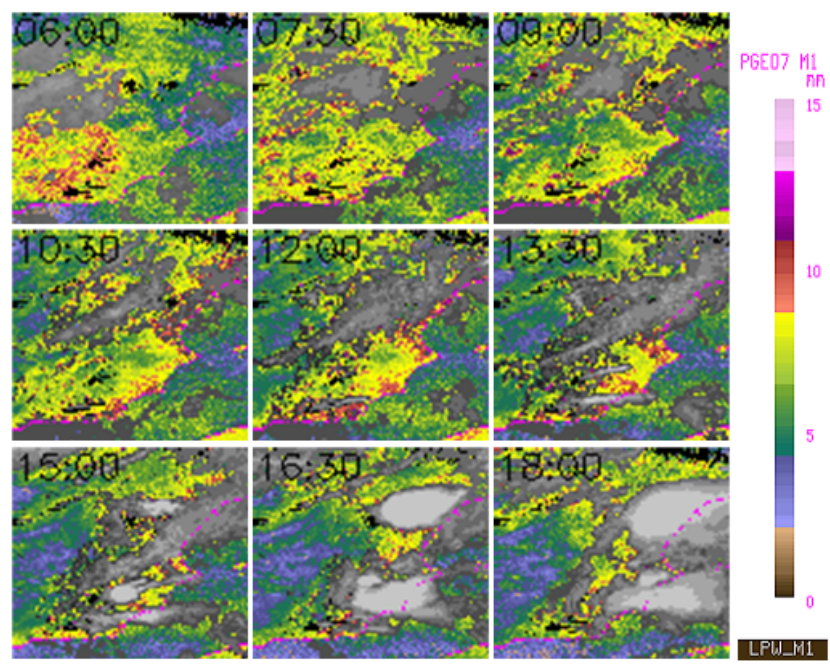

Figure 5. M1 evolution from 06:00 to 18:00 Z every 1:30 h. The colour scale has been enhanced in order to allow a better appreciation.
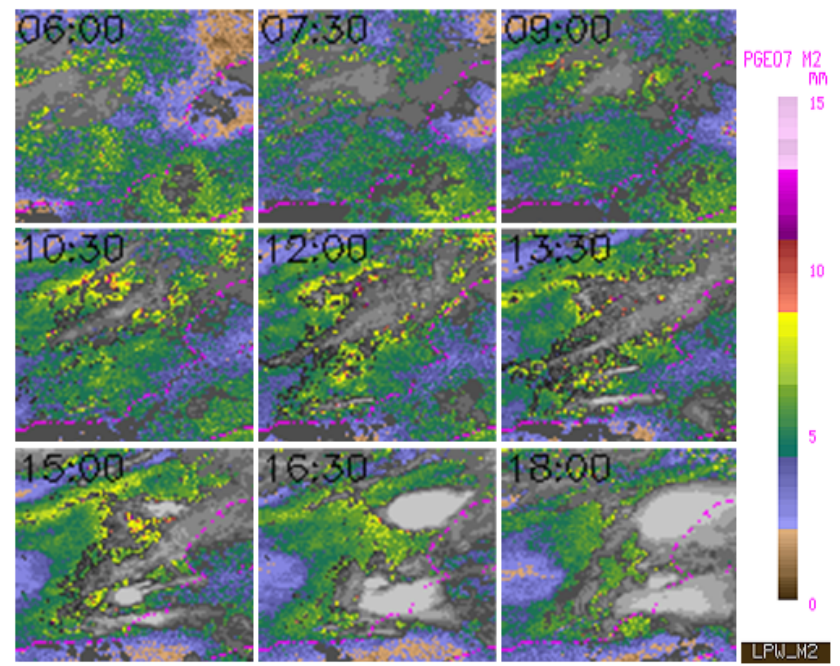

Figure 6. M2 evolution from 06:00 to $18: 00 \mathrm{Z}$ every 1:30 h. The colour scale has been enhanced in order to allow a better appreciation.

MODIS AQUA at 12:30 Z. The M2 pattern fits better the analysis at 12:00 Z than the forecast (above) and is in agreement with MODIS pattern, though the latter is slightly wetter since the considered layer is somewhat thicker. Similar results were found for the total layer parameter, always showing better agreement with the analysis as expected.

The comparison for the LI parameter (not shown here) confirmed these findings, presenting good agreement of patterns, being closer to the analysis than to the $00+12 \mathrm{H}$ forecast.

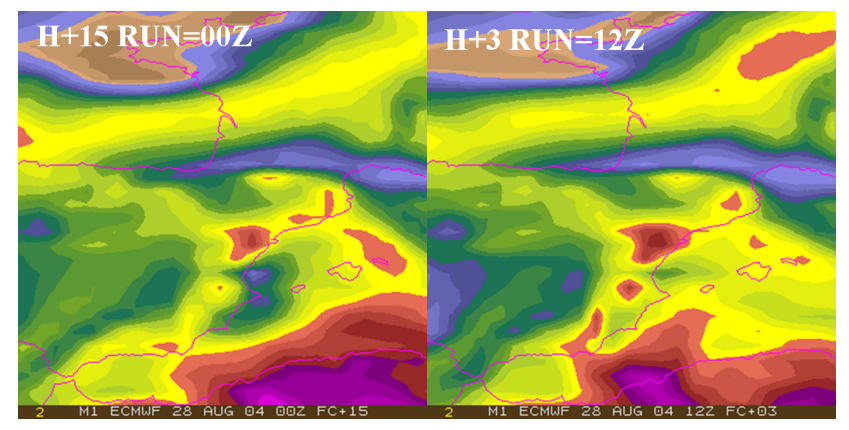

Figure 7. M1 parameter derived from ECMWF forecasts for the $00: 00 \mathrm{Z}$ run $+15 \mathrm{H}$ (left) and for the $12: 00 \mathrm{Z}$ run $+3 \mathrm{H}$ (right).
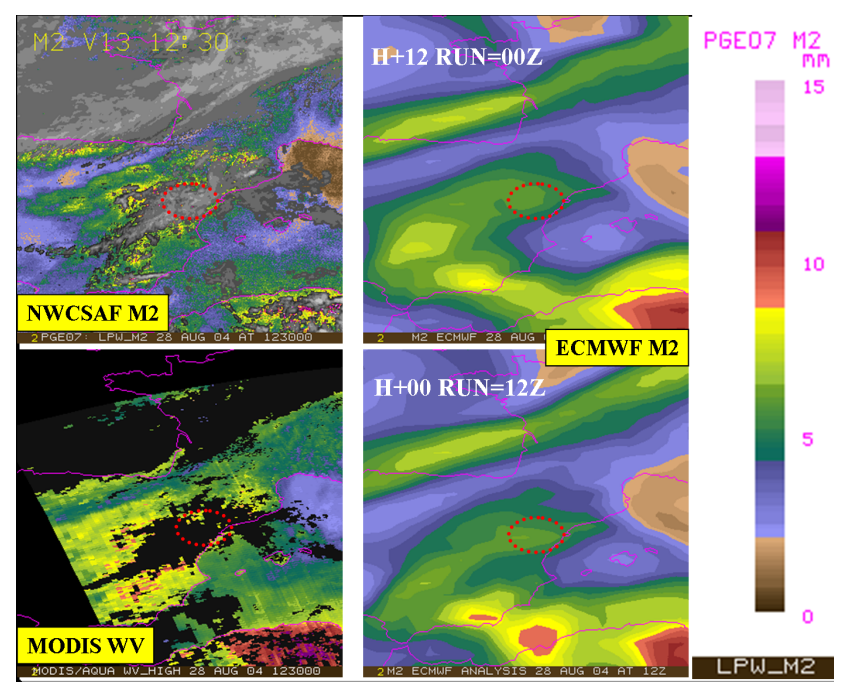

Figure 8. Comparison of M2 NWCSAF parameter (up left) with the M2 derived from the ECMWF 00:00 Z run $+12 \mathrm{H}$ forecast (up right), the 12:00 $\mathrm{Z}$ analysis (down right) and MODIS AQUA equivalent parameter WV_high: 700-300 hPa (down left)

\section{Conclusions}

Two neural networks were trained in order to provide additional vertical moisture information (850-700 and 700$473 \mathrm{hPa}$ ). Both parameters retrieved from IR SEVIRI channels gave information which is consistent with the ECMWF analyses. The middle levels spatial distribution of moisture presented a better agreement with the ECMWF analyses than with the $+12 \mathrm{H}$ forecast.

Humidity advections for M1 layer were clearly appreciated on the image loops thanks to the SEVIRI temporal resolution (15 min).

MODIS images (AQUA) available at that time were used to test the consistency of the spatial patterns. The new sublayer parameters showed a good performance, proving to be useful as a help in the detection of severe weather episodes. Further work in perspective is the testing of these new parameters in other case studies in order to analyse the humidity 
distribution in different situations, such as other summer season conventional convection events over Europe, as well as other different events, like the Gordon hurricane (2006). It is envisaged the possible inclusion of these new sub-layers in new algorithm versions. In any case, further algorithm development activities are also ongoing in the meantime (physical retrieval algorithm).

Acknowledgements. This study was carried out in the SAFNWC framework. The SAFNWC (constituted by Météo-France, SMHI, ZAMG and INM) is a co-operation agreement between EUMETSAT and INM.

The authors would also like to thank A. Conesa for the complete monitoring and thorough report of the event that he carried out.

Edited by: F. Stel and D. B. Giaiotti

Reviewed by: two anonymous referees

\section{References}

Conesa, A.: Tornado y Supercélula en Alfambra /Corbalán (Teruel), 28-Agosto-2004, http://www.spainsevereweather.com/ ver-reportaje.php?id=54, last access: April 2008.

Curtis, L.: Midlevel Dry Intrusions as a Factor in Tornado Outbreaks Associated with Landfalling Tropical Cyclones from the Atlantic and Gulf of Mexico, Weather Forecast., 19, 411-427, 2004.
Martínez, M. A., Velázquez, M., Manso, M., and Mas, I.: Application of LPW and SAI SAFNWC/MSG satellite products in preconvective environments, Atmos. Res., 83, 366-379, 2007.

Martínez, M. A., Velázquez, M., Cuevas, G., and Ruiz, J.: Improvements to the neural network retrieval of Layer Precipitable Water including an IR SEVIRI local radiancebias correction. Joint 2007 EUMETSAT Meteorological Satellite Conference and the 15th Satellite Meteorology \& Oceanography Conference of the AMS. Amsterdam, The Netherlands, http://www.eumetsat.int/Home/Main/Publications/ Conference_and_Workshop_Proceedings/groups/cps/documents/ document/pdf_conf_p50_s10_12_martinez_p.pdf (last access: April 2008), 2007.

Martínez, M. A., Velázquez, M., Cuevas, G.: Algorithm Theoretical Basis Document for "Clear Air Products" (TPW-PGE06 v3.0, LPW-PGE07 \& SAIPGE08v2.0), http://nwcsaf.inm.es/Scientific\%20documentation/ SAF-NWC-CDOP-INM-SCI-ATBD-06_v2.0.pdf (last access: April 2008), 2008.

University Corporation for Atmospheric Research (UCAR): Windows to the Universe, http://www.windows.ucar.edu/tour/link= /earth/Atmosphere/tornado/formation.html\&edu=high, last access: April 2008. 